\title{
College \& Research Libraries News INDEX TO VOL. 53 (1992)
}

\author{
Compiled by Eldon W. Tamblyn \\ Portland State University, Oregon
}

A

AACR2, news note, 567

Abbott, Lisa T., photo., 128

"Academic library public relations: using internal publications to spread the news," Bean, 91

Academic or Research Librarian of the Year Award, Baker \& Taylor, 1992, 184, 528; 1993, 527

"Academic reference service over electronic mail ${ }^{\text {" Bristow, 631-37 }}$

"Access technology in academic libraries," Lynch, 243-44

"Accreditation advisors," 449

AIDS, news note, 438

Acquisitions, 60-61, 126-27, 212, 275 $77,343-44,415,473-74,540-41$, $602-3,673,732-33$

Acquisitions (by author, subject, or $t i$ (le): Aboriginal justice, 344; Addams, Charles, 603; Adomeit, Ruth E. (children's books), 212; Affleck, Thos. (agriculture), 415; Africa (Laurence), 733; Agriculture (Affleck), 415; Architecture (Howe), 541; Auerbach (Herbert S.) Coll., 540-41; Autographs, 344; Automobiles (Clark), 275; Barbarian Press, 474; Beckwith-Fox family, 673 ; Boethius, 603; Botany, 602-3; Bristol phoenix, 540; Brooke-Rose, Christine, 415; Brown, Hugh N (PR), 61; Carter family (Va.), 343; Cartoons, editorial, 603: Cather Willa, 344; Cheney, Nan, 474; Cherokee Nation, 61; Chicago gay \& les bian newspapers (microfilm), 474; Children's books, 733 (Adomeit, 212; Sugarman Award, 415); Clark, Henry A., Jr. (automobiles), 275 Clerk's Press, 732-33; Commins, Saxe, 603; Crime broadsides (Reni- er, 19th c.), 415; De structura ora tionis, 344; Dickinson, Emily, 540; Douglas, Fenner (organ hist), 276 Douglas, Stephen A., 61; Drama, 16th-18th C. 415; Editorial cartoons, 603; Elliott-Davies Coll. (Pacific NW Americana), 733; Farm diary (Wood), 276-77; Fasting girls, 127; Firestorm, East Bay, Calif, 541: Fitzgerald, Robt., 343; Flanigan (Alexander). Hymnology Coll., 540 Fore-edge painted books, 344; Fowles, John, 126; Gay \& lesbian newspapers (microfilm), 474; Germany, 541; Gissing, Geo., 415; Grant, Ulysses S., 61; Hardwick, Elizabeth, 344; Hobhouse, Janet, 673: Homosexual newspapers (microfilm), 474; Howe, John H., 541; Huxley, Aldous, 276; Hymnology (Flanigan), 540; Illustrated books, 603; Japanese lit., 276; Judaica, 733; Justice, aboriginal, 344; Kant, Immanuel, 126; Kornitzer, Bela, 61; Laurence, Margaret (Africa), 733; Law, rare books, 342. Lewis \& Clark (1807), 344; Lincoln, Abraham, 61; Lish, Gordon, 275-76; London, Jack, 540; Lowell, Robt., 344; Maliseet people (Paul), 276; MSS, 212; Massell, Sam, 126; Melville, Moby Dick eds., 673; Michener, James A., 603; Microfilm (Chicago gay \& lesbian newspapers), 474; Mosher, Chas. A., 61; Music, arrangements, 540; Natl. Women's Political Caucus, 61; Nazareth Coll. Lib. 673, Near East, 733; Odets, Clifford, 344; Organ hist. (Douglas), 276; Orwell, Geo., 1984 MS., 673; Outcault, Richard F., 474; Pacific NW Americana (Elliott-Davies), 733; Paul, Pe-

FIING is word-by-word

ARRREVIATIONS: Standard abbreviations are used except in titles. Names of some organizations, ALA, ACRL, LC, etc., are also abbreviated and are alphabetized as if spelled out Special abbreviations:

$\begin{array}{ll}\text { appt. } & \text { appointment } \\ \text { f. } & \text { foundation } \\ \text { port. } & \text { portrait } \\ \text { prof. } & \text { profile } \\ \text { prog. } & \text { program }\end{array}$

SPECIAL USAGES: More than one reference per page is indicated in parentheses. Under the heading "Acquisitions (by author, subject, or title)" parentheses may enclose donors' names (for subjects) or subjects (for named collections). ter (Maliseet people), 276; Pennyroyal Press, 126-27. Pforzheimer Coll., 415; Photos., East Bay firestorm, 541; Plays, 16th-18th c., 415; Poland, hist., 474; Poles in America, hist., 474; Polish-language books, 474: Polish lit. 474; PR (Brown), 61; Rare books, 212 (law, 342; 16th c., 344); Renier crime broadsides (19th c.), 415; Robinson, Earl, 733; Russia, 541; Shakespeare, folio (1623), 126; Shaw, G. B. (Albert), 60-61, 212; Sinclair, Upton, 276; Smith (Sylvia) Archives, 61; Spain, Civil War, 733; Széchényi family ports., 603; Timberlake, Clare H., 126; Tourel, Jennie, 415; Villard, Henry S., 473-74; Wedgwood, Josiah, 473; Welk, Lawrence, 540; Wells, H. G., 733; Weltner, Chas., 126; Welty, Eudora, 733; Wilbur, Crane, 474; Witelo, Optics, 344; Women's studies (Ms. F. for Women), 733; Wood, Dave (farm diary), 276-77; Wright, James Arlington, 673; "Yellow kid," 474; The Young uoman's guide to virtue . . , 127

Acquisitions (by institution): American U., 342; Amherst Coll., 540; Atlanta Hist. Soc., 126; Baker Coll., 673; Birmingham Mus. of Art, 473; Boston U., 126, 415, 473-74; Brown U., 60-61, 212, 673: Chicago Hist Sac. 474; Col. Williamsburg F., 343; Dartmouth Coll., 673; Drew U., 61; Folger Shakespeare, 602-3; Ford (Henry) Mus., 275; Geo. Wash. U., 415; Harvard Law School, 415; Huntington, 540, 673; Ind. U., 212, 275-76, 344, 415; Ind. U.-Purdue U. of Indianapolis, 61; Kent State U., 344; LC, 733; McMaster U., 733; NYPL, 541, 603; N. D. State U., 540; Oberlin Coll., 61, 276, 733; Ohio State U., 415, 603; Princeton U., 603; Radcliffe Coll., 61; Redwood Lib. \& Athenaeum, 603; Reformed Theology Seminary, 540, Roger Williams U., 540; Rosemont Coll., 733; Rutgers U., 673; Southern III. U., 61; Syracuse U. 344, 474; Tex. A\&M U., 415; U. of Akron, 61; U. of B. C., 474; UC (Berkeley, 540-41; Santa Cruz, 344); UI, U-C, 733; U. of Manitoba, 344; U. of Mich-Dearborn, 276; U. of Minn., 541, 673; U. of Neb.-Lincoln, 126-27, 344; U. of N. B., 276; U. of Pittsburgh, 474; U. of Southern Cal- 
if 474; UT, Ausrin, 126, 344, 415 603; U. of Toledo, 732-33; U. of Tulsa, $61 ; \mathrm{U}$. of Va., 212; U. of Wash., 733; U. of Wis.-EC, 276-77; Vanderbilt U., 127; Wash. U., 603; Whitman Coll., 733; Yale U., 343

Adams, Charles Marshall, deceased, 350

Adams, Mignon, "The role of academic libraries in teaching and learning," 442-45

Advances in preservation and access, news note, $502-3$

Africana Librarians Council, news note, 567

Airth, Elizabeth J., retired, 737

Albritton, Rosie L., "Doctoral Dissertation Fellowship awarded" \& port. 279

Aleksandrovich, Vladimir, Grand Duke, port., 541

Alire, Camila A., "WHCLIS teleconference stimulates action plan for Colorado," 105

Alldredge, Noreen S. "Coalition for Networked Infomation: the second year," (part 1, 10-11; part 2, 98-99); "Network navigating and navigators." 399-400

Allen, Beverly E., news note, 131-32

Altschiller, Donald, "Read any good (suggestion) books lately?" 92-93

Alvin David photo 274

Alvin, Glenda, photo., 280

Amer. Antiquarian Soc, news note, 567

AAHE, "Information literacy topic at AAHE spring meeting," 126

ALA, "April 15 deadline for library/ book fellows," 274

ALA, ASCLA, news note, 275

ALA, Black Caucus, "Culture keepers: enlightening \& empowering communities," 638-39

ALA, "Call for America's Libraries," news note, 212

AIA, Code of ethics, news note, 567

ALA, Council, news note, 501

ALA, "Help available for locating copyright holders," 125

ALA, IRC/IRRT, "Guidelines for international visits available," 60

ALA, IRC/IRRT, JCIE, news note, 630

ALA, "Journey to Transylvania: the 1991 ALA colloquium on library science," 172-75, comment, 324

ALA, "Media tips:" ("how to get speaking opportunities," 127; "speaking successfully," 182; "what to do when the media calls and some quotes your audience will love," 326-27)

ALA, news note, 37475

ALA, OIF, response to letter to the ed. "Rust support evil?" (52:738) comments, 207, 265

ALA, "Products available for Black history month, "60-61

ALA, PIO, "National Library Weekmaking it happen," 88-89

ALA, "Reaching out to the media, 259

ALA, Washington Office, news note, 501-2

ALA, "Your responsibilities as a media spokesperson," 408

"AIA candidates share their plans for the future," 249-54

"ALA seeks tibraries for Arthurian ex hibits," 503

Ameritech, news note, 209
Anders, Mary Edna, news note, 542

Anderson, Susan, photo., 280

Andersson, Ellen, retired 133

Annenberg/CPB project, news note, 440

"Annual Conference: meetings for all minds," ACRL, 328-35, 412, 517-22 575-77

Antonetri, Martin, Nijhoff award, 389

Appointments, 63-65, 132-33, 217-18 282-84, 346-48, 416-18, 475-78, 543 $44,604-7,674-76,734-37$

"Are standards appropriate?" Pierson 453

Art Libraries Soc of $\mathrm{N}$. America "Documentation of performance art," 246-48

ALCTS, "New AV guide available," 278

ACRL, ASC, "Model statement for the screening and appointment of academic librarians using a search committee" 642-45; "Standards for faculty status for college and university librarians, ${ }^{n} 317-18, \mathrm{com}$ ment, 453

ACRL, APGTF, "Guidelines for the preparation of policies on library access," draft, 709-18

ACRL, "And the winners are " 465-67

ACRI, Annual Conference, 1992, $328-35,412,517-22,575-77,655$

ACRL, Annual Report, 1991-92, 64764

ACRL, BIS, BIS Bibliographic Instruclion Publication of the Year Award, 1993,532

ACRL, BIS, "BIS members: turn in survey," 270

ACRL BIS, Miriam Dudley Bibliographic Instruction Librarian of the Year Award, 1992, 279-80; 1993. 527-28

ACRL, BIS, news note, 502

ACRL, BIS, TFSOPE, news note, 695

ACRL, BIS Teaching methods comm., news note, 565

ACRL, Board of Directors, "Highlights" (Jan., 176-78; June \& photos. 524-25); photo. \& roster, 1991-92,661

ACRL, Budget \& Finance Comm., "Financial report," 662-64

ACRI, "Candidates for ALA Council"" 255-57

ACRL, "Candidates for 1992 elec tions," 18-20

ACRL, Dep. Exec. Director, photo., 382

ACRL, EBSS, BIEC, "Information retrieval and evaluation skills for education students," 583-88

ACRL, EBSS, news notes, 696 (2)

ACRL Exec. Director, "Letter from the executive director" \& port., 652-53; photo., 382

ACRL, ECLSS, "Bylaws," 262-65

ACRL, "Guidelines for the prepara tion of policies on library access," draft, 709-18

ACRL, Hugh C. Atkinson Memorial Award, 1992, 279; 1993,528

ACRL, ILAB, "Information literacy experts sought," 207; "Need help with your information literacy program?" 718

ACRL, Midwinter Meetings, 1992, 13 . 1993, 721-27

ACRL, "Mission," 648

ACRL, "Mousepad" \& photo., 375
ACRI National Conference 1902 $27-53,100-101,186-87,203,376-85$ $455-60,591$

ACRL, National Conference, 1992 ("An invitation to Salt Lake City...." 27-53; "A potpourri of practical ideas," 455-60; "Proceed ings now available!" 591; "Research and academic librarians: a global view," 203; "Salt Lake Ciry-Culture and entertainment," 100-101; "Sal Lake City-restaurants and clubs to enjoy," 186-87; "Spring and diversi ty in the air at ACRL conference," 376-85)

ACRL, "New ACRL publications," 318

ACRL, "New BI book published, " 274

ACRL, "New titles from ACRL in 1992 , 660

ACRL, "New women's studies book published," 375

ACRL, "Official ACRL documents," 107

ACRL, Past-President, photo., 382

ACRL, President, "Media tips: how to get speaking opportunities," 127 "Message" \& port., 649-50; "Noth ing ventured, no one gained" (52:640-41), comment, 207; pho tos., 379, 382, 528; "Recruiting the best and the brightest" \& port. 320-23

ACRL, PEC, "Education for profes sional academic librarianship," 590

ACRL, REDC, "Let ACRL's Racial \& Ethnic Diversity Committee know about your activities," 104

ACRL, RBMS, Katharine Kyes Leab \&

Daniel I. Leab American Book Prices Current Awards, 1992, 281 1993, 531-32

ACRL, RBMS, "33rd RBMS preconference to be in Santa Cruz," 310

ACRL, "Recruitment open for two ACRI editorships," 183

ACRL, STS, "Call for sci/tech abstracts," 254

ACRL, STS, Eunice Rockwell Oberly Award, 1993, 311, 532

ACRL, STS, FSTLRC, news note, 629

ACRL, TFWHCLIS, "Using White House Conference recommenda tions," 22, 25

ACRL, "The time to lead," 256-57

ACRL, Vice-President, "Message from the vice-president" \& port., 651 photo. 382

ACRL, WESS, Martinus Nijhoff International West European Specialis Study Grant, 1992, 389; 1993, 529 31

ACRL, WSS, "Compiles list of publishing opportunities, ${ }^{n}$ 274; news note 696

ACRL, "The year in review," 654-60

"ACRL approval plans," 448

"ACRL awards for 1993," 527-32

"ACRL Board actions" (Jan., 176-78; June \& photos., 524-25)

ACRL chapters. See Chapter topics

"ACRL chapters provide forum for electronic information discussions," Junion-Metz, 16-17, comment \& response, 265

"ACRL discontinues telephone jobline," 440

"ACRL discussion groups," 332

"ACRL honors outstanding contribu-

tions to academic librarianship," 279-81

"ACRL journals," 659 
"ACRL meetings in Denver," 721-27

"ACRL meetings in San Francisco," 330-35

"ACRL membership statistics," 650

"ACRL president's program \& reception," 328

"ACRL programs in San Francisco," 328-29

"ACRL publications," 318

ACRL publications in librariansbip, "Recruitment open," 183

"ACRL publishes statistics," 273

"ACRL seeks nominees for office," 256-57

"ACRL staff," 653

"ACRL thanks colleagues," 385

"ACRL wants you!" 592-95

ARL, news note, 504

Atkinson (Hugh C.) Mernorial Award, ACRL, 1992, 279; 1993, 528

Auburn U., news notes, 439-40, 504

Auer, Margaret E., appt., 604; news note, 475

Avram, Henriette D., news note \& port., 132

Awards, $184,279-81,311,346,375$, $389,438,440,527-32,568-70,596$, 656-57

Bailey, Robert G., deceased, 134

Bain, George W., "What if we viewed library instruction like driver ed?" 581-82

Baker, Anne Marie, news note, 604

Baker, Betsy K., "Named Dudley Bibliographic Instruction Librarian" \& port., 279-80

Baker, Therese D., "Request forms for extended campus library services," 260-61

Baker \& Taylor, Academic or Research Librarian of the Year Award, $1992,184,528 ; 1903,527$

Bales, Ted, acknowledgment, 236

Balinsky, Deborah Perkal, deceased, 608

Balsino, Vita, photo., 528

"Banned Books Week," news note, 503-4

Barker, Richard T, deceased, 134

Barnes, Roy E., retired, 478

Barry, James $W$., deceased, 218

Bartley, Linda K., news note \& port., 416

Basu, Patricia Lyons, appt., 605

Bates, John, "Private treasures in a public library," 541

Baumgartner, Robert W., retired, 676 Bean, Gladys Hyde, deceased, 134

Bean, Rick, "Academic library public relations: using internal publications to spread the news," 91

Beaubien, Anne K., "Media tips: how to get speaking opportunities," 127 ; "Message from the president" \& port, 649-50; "Nothing ventured, no one gained" (52:640-41), comment, 207; photos., 379, 382, 524 $25,528,661$; "Recruiting the best and the brightest" $\&$ port., 320-23

Begg, Karin E., photos., 524-25, 661

Benefiel, Candace R., "Fun, friends and good p.r.: celebrating National Library Week in an academic library," 85-87, 89

Bentley, Stella, port., 28

Berea Coll., news note, 437-38

Bergman, Sherrie $\mathbf{S}$., appt. \& port., 282 ; port., 28

Berry, John W., prof. \& port., 131
Beverage, John, retired, 418-19

"Beyond the book review," Thompson, 646

Bibliothèque de France, "The très grande bibliotheque and the library of the future," 394-96

$\mathrm{BN}$, "Toward a Bibliothèque Nation ale des Arts," 247-48

Biggs, Mary, letter to the ed., 265

Birdwhistell, Terry, news note, 63

BIS Bibliograpbic Instruction Publica tion of the Year Award, ACRL, BIS 1993,532

"BIS members: turn in survey," ACRL BIS, 270

Bishop, David F, appt. \& port., 416-17

Bishop, Dorcas M., deceased, 65

BCAIA, "Culture keepers: enlightening \& empowering communities, 638-39

Bodling, Kurt A., Rev., appt., 282

Boissé, Joseph, photos., 35, 379, 382 port 28

Bolt, Nancy, news note, 132

Bond, Julian, "About the cover" 371 (photo., cover, no. 6); port., 32

Bonk, Joyce J., retired, 544

Book reviews, 57. See also New publications

"Books are for use: Ranganathan centennial year," Shama, 502

Booth, Robert, deceased, 545

Borgman, Christine L., news note, 604

Borko, Harold, retired, 478

Boston Coll., "Reference roving at Boscon College," 634-37; "When are library records confidential?" 129, clarification, 276

Boucher, Virginia, retired, 284

Bourdon, Cathleen, photo., 382

Bowles, Garrett, news note, 345

Bowling Green State U., news note 210

Bowman, Martha, photo., 35

Bozeman, Patricia, letter to the ed. 104

Bradley, Kate, letter to the ed., 20

Bratton, Phyllis Ann K., letter to the ed. 411

Bregman, Adeane, "Reference roving at Boston College," 634-37

Breyfogle, Robert, retired, 607

$B Y U$, news note, 310

Bristow, Ann, "Academic reference service over electronic mail," 631-37

Brown, Barbara, "Preservation news" (52:649), correction, 6

Brown, Barbara, "Preservation news," 106

Brown, Claire, retired, 133

Buchanan, Paul, prof. \& port., 215

Buck, Anne M., prof. \& port., 63

Bucknell U., "Beyond the book review," 646

Burckel, Nicholas C.. "Electronic dissemination of full-text journal articles," 322-23

Butcher, Karyle S., "User statistics for CD-ROM selection and management." 505-6

Butler, Evelyn, deceased, 419

Butler, Meredith, news note, 63

Byrne, Sherry, "Preserving the nation's intellectual heritage: a synthesis," 313-15

Caldwell, John C, retired \& port. 607

CEMA, "Collecting primary materials of major ethnic groups." 157-59
"Call for America's Libraries," AlA news note, 212

"Call for sci/tech abstracts," ACRI STS, 254

"Candidates for ALA Council!" ACRL 255-57

"Candidates for 1992 elections ACRI, 18-20

Cardell, Victor, news note, 345

"A career in Bl: dead end or stepping stone?" Newby, 178

Cargill, Jean, news note, 278

Carpenter, Catherine, retired, 133

Carter, Catherine, retired, 348

Carvaho de Miranda, A., port., 203

Casorso, Tracy M., "Research materials: now only keystrokes away" \& photo., 128

"Cassettes of Annual Conference pro grams available," 522

Chadwyck-Healy, news note \& photo. 275

Chapter topics, 16-17 (comment \& response, 265)

Chapter topics ("ACRL chapters provide forum for electronic informa tion discussions," Junion-Metz, 16 17 comment \& response 265 )

Charlot, "Motherly care," 234 (photo. cover, no. 4)

Chisholm, Clarence Edward, de ceased 8 port., 676; port., 29

Chisholm, D. Hugh, retired, 419

Cboice, news note, 629

Christensen, Beth, news note, 345

Christiansen, Dorothy, prof., 131

Christie, Manson \& Woods, Rare Books and Manuscripts Librariansbip Auard, 1993, 532

Church, Virginia, retired, 284

Ciarfella, Bobbi, letter to the ed., 411

CUNY, "Gay studies center established," 210

Clarifications, 6, 276, 520

Clarke, Robert, appt. \& port., 417

Cleveland State U., "The CologneCleveland librarian exchange," 390 93; news note, 210

CNI, "Network navigating and navigators," 399-400; news note, 309

"Coalition for Networked Informa tion: the second year," Kirk, part 1 10-11; part 2, 98-99

Cobb, David, appt., 282

Cochran, Richard M., appt., 734

Cochrane, Lynn Scott, appt. \& port. 543; "Using White House Conference recommendations, "22, 25

COLLDV-L, news note, 565

"Collecting primary materials of ma jor ethnic groups," Dowell, 157-59

CERL news, "Feature your collection on a CERI news cover, "106, 570 "In the news," 4, 84, 156, 236, 308 $372,436,500,564,628,692$; "June 2 deadline for classified ads," 311 listserv news note, 567; "Put CERI news on your mailing list," 101 , 175, 408; "Share your library's news," 447; "Share your opinion with CERL news readers," 14, 97, 174; "Statement of ownership and management," 667; "Witty suggestion book comments sought, 93 "CGRL news: breath of fresh air," Woodall, 535

"CERL news guidelines for submis sion of articles or columns," 26

"CERL news reporters needed," 41, 91,187

"CERL news staff member has a win- 
ning idea," 89

"The college library as guinea pig." Hayes, 572-74

"The Cologne-Cleveland librarian exchange," Peters, 390-93

Columbia U., news notes, 314, 565

Comaromi, John P., deceased, 218

Community College Learning Resources Achievement Awards, EBSCO , 1992, 280-81; 1993, 528-29

Condron, Lyn, "Make the most of your student assistants, " 5-6

Conference circuit, $246-48,322-23$ $376-85,394-96,399-400,402-3,455$ $60,638-39,640,707-8$

Conference circuit ("Culture keepers," Delacroix, 638-39; "Documentation of performance art," Murphy, 246-48; "Earlham's BI enhances teaching and learning, "Hardesty, 402-3; "Electronic dissemination of full-text journal articles," Burckel, 322-23; "Network navigating and navigators," Alldredge, 399-400; "New Delhi, India, hosts librarians from saround the world" Ford, 640 "A potpourri of practical ideas from ACRL's 6th National Conference, 455-60; "Shifting paradigms for li brarians," Hubbard, 707-8; "Spring and diversiry in the air at ACRI conference," 376-85; "Toward a Bibliothèque Nationale des Arts," McKee, 247-48; "The Très grande bibliotheque and the library of the future," Oberg, 394-96)

Cook, Jean G., retired \& port., 218

"Coping with repetitive-motion injuries in a large academic library," Eissinger, 161-62, comment, 412 $\mathrm{CPB}$, news note, 440

Corrections, 6, 276, 520

Crampon, Jean $\mathrm{E}_{-1}$ letter to the ed. 412

Crawford, Susan, news note, 475

Crismond, Linda, news note \& port.

373; news notes, 437, 501; photo. 376

Cronin, Blaise, news note, 63

Crooks, James E., "UC tries FirstSearch, " 464

Crowe, William A, news note, 604

Cruzat, Gwendolyn S., retired, 607

"Culture keepers: enlightening \& empowering communities," Delacroix 638-39

Cunningham, Joseph C., retired, 544

Curtis, Peter Harris, news note \& port., 542

D

da Conturbia, Sandra, "Fun, friends and good p.r.: celebrating Nationa Library Week in an academic library, " 85-87, 89

Dana (John Cotton) Library Public Relations Award, Wilson ( $\mathrm{H}$. W. Co, 1992, 568-70 \& cover; 1993 $438,570,596$

Davis, Joyce, appt. \& port., 543

Davis, Mary Ellen $K$. "In the News," $4,84,156,236,308,372,436,500$ $564,628,692$

Davy, Ed, retired, 676

"Death of the book greatly exaggerat ed," Young, 452-53, comment, 535

Deaths, 65, 134, 218, 284, 350,419, $545,608,676,737$

de Klerk, Ann, "Electronic publishing and networking '92," 181-82; letter to the ed., 535-37
Delacroix, Sheila, "Culture keepers enlightening \& empowering communities, "638-39

Delauche, Jean E., port., 29

Dennis, Rodney, retired, 133

DePaul U., "Acadenic library public relations: using internal publica tions to spread the news," 91 ; news note \& photo., 696

Diablo valley Coll., John Cotton Dana Library Public Relations Award, 568-70

Dillon, Dennis, "The future of reference IV: a response " 513-14

Dilmore, Don, appt., 675

Di Trolio, Trudi, "Using a checklist for library staff orientation and training;" 695

Doctoral Dissertation Fellowship, ISI, 1992,$279 ; 1993,529$

Document delivery and on-demand publishing: implications for reference service," Eaton, 508-10, re sponses, $512-14$

"Documentation of performance art, Murphy, 246-48

Dornin, May, deceased, 350

Dowell, Connie V., "Collecting primary materials of major ethnic groups," 157-59

Downing, Arthur appt., 475-76

Doyle, Marion, deceased, 218

Doyle, Robert P. "Journey to Transylvania: the 1991 ALA colloquium on library science," 172-75, comment, 324

Drake, Miriam A., "Named winner of Atkinson award" \& port., 279

Dubin, Eileen, photos, 524-25, 661

Dudley (Miriam) Bibliographic Instruction Librarian of the Yea Award, Mountainside Pub. Co. 1992, 279-80; 1993, 527-28

Duffett, Gorman L.-appt, 605

Dumont, Paul E., "Gets award for leadership in community colleges \& port., 280-81

Dunlap, Abbot Fidelis James, de ceased, 350

Dunlap, Ellen S., appt. \& port., 476

\section{E}

“Earlham's BI enhances teaching and learning," Hardesty, 402-3

East, Mona, deceased, 737

Eaton, Nancy L, "'The future of reference IV: document delivery and on-demand publishing: implications for reference service," 508-10

Eberhart, George M., "New publications." 54-56, 108-12, 205-6, 271-72, $340-41,409-10,470-72,599-601,670$ 71, 730-31. See also Book reviews

Eberhart, George M., "Publicarions" (52:656), comment, 20

Eberhart, George $\mathrm{M}_{\text {., The whole li }}$ brary handbook, rev. of, 57

EBSCO, Community College Learning Resources Achievement Awards, $1992,280-81 ; 1993,528-29$

EBSCO news note, 501

Eckerd Coll., "Earlham's BI enhances teaching and leaming, ${ }^{\text {" 402-3 }}$

Eckert, Robert, retired, 65

"Education for professional academic librarianship," ACRL, PEC, 590-91

"ERIC holds focus groups," 273

EDUCOM, "Shifting paradigms for librarians," 707-8

$\mathrm{Ei}$, news note, 309-10

Elbaz, Sohair, appt. \& port., 543
Elder, Nancy, "The future of reference IV: a response," 512-13

"Electronic dissemination of full-text journal articles," Burckel, 322-23

"Electronic publishing and networking '92," de Klerk, 181-82

Elsbernd, Mary Ellen, port., 29

"Emory University Libraries' Environ mental Action Force, ${ }^{n}$ Wagner, 579 80

Erickson, Alan, retired, 133

Erickson, Ture, retired, 348

Essinger, Richard A., "Coping with repetitive-motion injuries in a large academic library," 161-62, comment, 412

Eunice Rockwell Oberly Award, ACRL, STS, 1993, 311, 532

Euster, Joanne R., appt, \& port. 476

Evans, Sally Romer, retired, 607

Exchanges, 390-93, 630

Farber, Evan Ira, news note, 542; photos. 35, 403, 524-25, 661

Faxon, news note, 501

"Feature your collection on a CERL news cover," 106,570

Feller, Siegfried, retired, 133-34

Ferran, "Vista de S. Francisco," 306 (photo., cover, no. 5)

Ferrell, Mary Sue, photo., 525

"A few words on our type," Morris, \& response, 324

Field, Phyllis, news note \& photo., 694

"First multicultural library intems named," SUNY, U. at Albany, 60

"Food for thought," Gater (52:711) comment, 104

"Food nor only possible break for readers," Viera, 104

Ford, Barbara J., "New Delhi, India, hosts librarians from around the world," 640; photos., 382, 524, 661

Forro, Denise, "Patron access to on order and in-process titles," 702-8

Fox, James, appt. \& port., 417

Frank, Dennis, letter to the ed., 411

Franklin, Hardy, "AI.A candidates share their plans for the future" $\&$ port., 249

Friends of Libraries USA, news note, 374́-75

Frost, John Eldridge, deceased, 608

Fulbright Scholar Program, news note, 274

"Fun, friends, and good p.r.: celebrat ing National Library Week in an ac ademic library," Benefiel, 85-87, 89 "The future of reference IV:" ("document delivery and ondemand publishing: implications for reference service" "Eaton, 508 10; "a response," Dillon, 513-14; "a response," Elder, 512-13)

Garrison, Guy, news note, 604; retired \& port. 348-49

Gaskell, Carolyn, "Optical local area network at Walla Walla College, 112

Gater, Helen L., "Food for thought" (52:711), comment, 104

"Gay studies center established," CUNY, 210

Geraci, Diane, "Optical scanning in an academic library, "698-701

"Get some better looking librarians (and other suggestions from students)," 467 
Goldberg, Cynthia, news note, 674 Goldberg, Marty, letter to the ed. 412 Goldsmith, Benjamin, "Private treasures in a public library," 541

"Good acting secret of successful BI," Jacobson, 441, comment, 639

Gotwals, Joan i., news note, 674

Grants, 62, 127-30, 214, 277, 342-43 $413,414-15,473,539-40,602,672$ $73,696,732$

Grants (by grantee): Adams State Coll. 672; Adrian Coll., 672; ALA 62 Amer. School of Classical Studies, 62; Amer. U., 342; Amhers Coll., 127; Ariz. Státe UJ, 277; Bard Coll., 127; Broadcast Pioneers, 62 Brown U., 473; CRL comission: 51:144, 465, 780, 990); Columbia U., 214, 414; CIC, 214; Cornell U. 732; DePaul U., 127-30, 696; Dra benstot, Karen, 414; Drexel U. 414; Duke U., 62, 539; Eckerd Coll., 539; Emary U., 130; Folger Shakespeare, 62, 130, 539; Hahnemann U., 672; Harvard U., 62, 277; Holston Academic, 732; Hoover Inst., 277; Ind. U., 214, 342 (3) 732; John Carter Brown, 130; KSU 414 (2); Laramie Co. Community Coll, 672: Lib of Mich. F 602. Lincoln U., 62; McMaster U., 62; Mont State U., 539; NYPL, 602; NYS Newspaper Project, 62; Niagara U., 214; NCSU, 130, 539; NEDCC, 672 Northern Ill. U., 473; Oberlin Coll. 214; Pa. State U, 214, 342; Re deemer Coll., 414; Reed Coll., 672 RLG, 214, 342-43; Rice U., 277, 732 Rutgers U., 473; St. Bonaventure U., 130; St. John's Coll, 672; St. John's U., 62, 343; San Jacinto Coll Central, 62; Smithsonian Inst., 602 SMU, 343; Stanford U., 414; State U. Colleges, Buffalo, $342 ;$ U. o Ala., 672-73; U. of Ark., Little Rock 130; U. of Calgary, 732; UC (Irvine, 473; Santa Barbara, 130; Santa Cruz, 414); UI, U-C, 130; U. of Ind. 130; U. of lowa, 539; U. of Ky. 214; U. of Manitoba, 62, 214, 473 $\mathrm{U}$. of Md. Health Sciences, 277; U of Mich., 414; U. of Mont., 214; U. of Nev. System, 62; UNC (Asheville, 62; Chapel Hill, 277, 539, 602 673): U. of N D 732, U of $\mathrm{Or}$ 602; UT (Arlington, 343; Austín 414-15, 539): U of Victoria 415: U of $\mathrm{Va}, 539-40$; U. of Wash., 602; U of Waterloo, 62; Warner, Amy, 414 Whitman Coll., 130, 277, 673; York $\mathrm{U}, 602$

Grants (by grantor): Abell-Hanger $F$ 343; Abrams (Talbert \& Leota) F., 602; Adrian Coll. Class of 92,672 AT\&T, 696: Bremer (Otto) F 732 Bruton (David Jr.) Charitable Trust 343; Cafritz (Morris \& Gwendolyn) F, 130; Canada, 214, 732 (Quebec, 732); Canadian Council of $\mathrm{Ar}$ chives, 415, 473. CSSHRC, 62 (2) 414, 732; CLR, 214, 539; Eckhardt Gramatté F. 214; Educational F. 277; Ferris (Booth) F., 214; Fiedler, Alice, 414; Fondren F., 62; Good man, Leonard S., 342; Grainger, Wm. Wallace, 130 ; Haag, Donald M., 214, Hale, H. D. \& Joyce, 414 Harris, Dorothy V., 342; Hewlett (Wm. \& Flora) F., 214; HEA, 62 $130,214,342,343,413,473,539-40$ (4), 602, 732; Hobby F., 277;
Houghton Mifflin, 62; Humana, 214; IIl. SL, 473; IBM, 673; Intl. Council for Canadian Studies, 732 Japan F., 473; Japan W. E. C. Fund, 214; Joint Comm. on Soviet Studies Lib. Backlog Prog., 342; Jonsson F. 130; Josey (John $H$. \& Carolyn Cobb) Endowment Fund, 672-73 Kodansha, Ltd., 602; LSCA, 130 672: Littauer (Lucius N.) F., 277, Luce (Henry) F., 277, 602, 732 McBirney, Ruth, 673; Mellon (Andrew W.) F., 414, 539; Mitchell Ruth C., 342; Murdock (M. J.) Char itable Trust, 672; NEH, 62 (2), 127 30 (2), 214, 277, 342-43, 414-15, $473,539,672,732$ (2); NHPRC, 62 NIH, 414; NLM, 277, 672, NY Newspapers F., 62; Ont. Council of Archives, 602: Or. Community F 672; Ottenbeimer Bros. F., 130 Richardson (Sid) F., 343; Rosenberg, Claude \& Louise, 414; Santa Cruz Co. Eamilies, 414; Shanahan Ilabelle 277. Smithson (James) Soc., 602; SSHRC(C), 62 (2), 414 732; State U. Colleges, Buffalo, USG, 342: Summerlee F., 343; Taishoff, Lawrence B., 62; Tex. Environmental Ctr. 732; Toshiba Intl. 473; Union Pacific F., 672; USDE $62,130,343,414$ (3), 672; Westing house Canada, 62

"Grants awarded to research libraries to provide national access to re sources," Loeb, 413

Greenfield, Melissa, photo. 280

Griffith, Belver, retired, 340

Griscom, Richard, news note, 345

Güereña, Sal, news note, 734

"Guidelines for international visits available," ALA, IRC/IRRT, 60

"Guidelines for the preparation of policies on library access," ACRI draft, 709-18

Gunnison-Wiseman, Deirdre, retired 134

Gupta, Usha, "Is FirstSearch really attractive?" 461-64

Gyeszly, Suzanne D., "Librarians meet in Budapest," 374

Hall, Anthony, retired, 65

Hall, Blaine H., "Salt Lake Cityculture and entertainment," 100 101; "Salt Lake Ciry restaurants and clubs to enjoy," 186-87

Hanson, Roger K., port., 29

Hardesty, Larry, "Earlham's BI en hances teaching and leaming, ${ }^{\text {4 } 402-3}$

Harper, Penney, "Spellcheckers must be checked," 567

Harvard U., KSG, "Read any good (suggestion) books Iately?" 92-93

Harvard U., news note, 209

Harwood, Judith, news note \& port. 345

Hastreiter, Jamie, "Earlham's BI enhances teaching and learning, "402-3

Hause, Aaron, deceased, 218

Havlik, Robert J., retired \& port., 544

Hay, Fred, news note, 278

Hayes, Sherman, "The college library as guinea pig," $572-74$

Heckatt, Ronald J., K. G. Saur Award 346

Hedberg, Jane, "Preservation news," $200,269,339,407,469,536,598$ $668-69,729$
Helm, Margie May, deceased, 350

Helms, Frank, news note, 734

"Help available for locating copyright holders," ALA, 125

Henderson, Carol C., "Washington hotline," 24-25, 189-90, 267-69 (comment, 453), 337, 405, 468-74, $534,597-603,666-69,728$

Henderson, David, "Earlham's BI enhances teaching and learning, "402-3

Henderson, Donald C., Jr., retired, 340

Herring, Mark, appt., 675

Hesslein, Shirley, deceased, 545

Hinojosa, Susana, "Racial \& ethnic di versity: information exchange, $270,338,537,669$

Hinshaw, Marilyn, news note, 734

Hogan, Sarah T., retired, 737

Holley, Edward G., news note \& port, 345

Holloway, joaquin, news note, 734

Homes, Nellie M. deceased 676

Horrell, Jeffrey, prof., 215

"How NREN can meet the needs of academic libraries," Wand, 705-6

Hubbard, Willis M., "Shifting paradigms for librarians," 707-8

Hugh C. Atkinson Memorial Award, ACRI, 1992, 279; 1993, 528

Hunter, Lora, photo., 280

Hyltoft, John, retired, 607-8

The importance of acting," Winne grad, 639

"In the News," Davis, 4, 84, 156, 236, $308,372,436,500,564,628,692$

Ind., news note, 694

Ind State U., news note, 620

Ind. U., "Academic reference service over electronic mail," 631-37; news note, 59-60

INFORMA, news note, 274-75

INFORMA 1992, news note, 440

"Information literacy experts sought," ACRL, ILAB, 207

"Information literacy topic at AAHE spring meeting," 126

"Information retrieval and evaluation skills for education students, ACRL, EBSS, BIEC, 583-88

Innovations, 91, 260-61

Innovations ("Academic library public relations," Bean, 91; "Request forms for extended campus library services," Baker, 260-61)

ISI, Doctoral Dissertation Fellowship, 1992,$279 ; 1993,529$

ISI, Samuel Lazerow Fellowship, 1992,$346 ; 1993,531$

IFLA, "New Delhi, India, hosts librarians from around the world," 640 ; news note, 210

ISOE, news note, 309-10

Irvine, Betty Jo, news note, 278

"Is FirstSearch really attractive?" 461-

"Issues raised by extended campus services librarians," Lessin, 14-15

J

Jacobson, Trudi E., "Good acting secret of successful BI," 441, comment, 639

Jenkins, Althea $\mathrm{H}$., "Letter from the executive director" \& port., 652-53; photos. 376, 382, 524-25, 661

Jermann, Peter, news note \& photo., 475

Job, Amy, news note, 345-46 
John Cotton Dana Library Public Relations Award, Wilson ( $\mathrm{H}$. W. $\mathrm{Co}$ 1992, 568-70 \& cover; 1993, 438 . 570,596

Johnson, Eric, Samuel Lazerow Fellowship, 346

Johnson, Guela, retired, 545

Johnson, Ralph, retired, 65

Jordan, Anne, retired, 284

"Journey to Transylvania: the 1991 ALA colloquium on library science, ${ }^{3} 172-75$, comment, 324

Julian, Charles A., appt., 734-35

Junion-Metz, Gail, "ACRL chapters provide forum for electronic information discussions," 16-17, comment \& response, 265

K

K. G. Saur Award for Best College $\varepsilon$ Research Libraries Article, 1992, 346; 1993, 532

Kaatrude, Peter B., appt. \& port., 543

Karlson, Marjorie, retired, 218

Katharine Kyes Leab \& Daniel J. Leab American Book Prices Current Awards, ACRL, RBMS, 1992, 281 1993, 531-32

Kathman, Michael, "The college li-

brary as guinea pig," 572-74

Kelly, Donna, photo., 280

Kent State U., news note, 565

Kilton, Thomas D., news note, 416

Kirk, Thomas G., ACRL vicepresident \& port., 465-66; "Coalition for Networked Information: the second year" (part 1, 10-11; part 2, 98-99): "Kirk and Wittkopf share their visions for ACRL" \& port., 194-99); "Network navigating and navigators," 399-400. "Periodicals in college libraries: are the challenges of rising subscription costs being met?" 94-97; photo. 525; port., 28

Knight, Helen, retired, 349

Koch, David, appr. \& port., 346-47

Kolb, Emery \& Ellsworth, photo. cover, no. 8

Koyama, Janice, news note, 215

Kozlova, Ludmila, port., 203

Kreitz, Patricia A., "Recruitment \& re tention in your own backyard," 237-40, comments, 411

Kreitzburg, M. J., letter to the ed., 104

Krumboltz, John, port., 517

"Kudos and complaints on Dead Sea

Scrolls coverage," Bozeman, 104

"Kudos for new CERL news format,

Ciarfella, Goldberg, Poole, 411-12

"Kudos for Selth," Kreitzburg, 104

"Kudos for Washington hotline,"

Scott, 453

L

LaBorie, Timothy, news note, 734

LaGrange, Johanne, "Fun, friends, and good p.r.: celebrating National library Week in an academic library," 85-87, 89

Landis, Linda, news note, $215-17$

Laney, Elizabeth, retired, 349

Lange, Marta, deceased \& port., 545

Larson, Jeffry, letter to the ed., 265

Lasater, Mary Charles, news note, 604

Laswell, Reg, retired, 349

LaVerdi, Adelaide, retired, 349

Lazerow (Samuel) Fellowship, ISI, 1992,$346 ; 1993,531$

Leab (Katharine Kyes \& Daniel J.) American Book Prices Current
Awards, ACRL, RBMS, 1992, 281. 1993, 531-32

Le Coeur Louis, "Fête du sacre et couronnement...," 434 (photo. cover, no. 7)

Lee, Hwa-Wei, news note, 674 ; news note $\&$ photo., 694

Lee, Sul $H_{\text {., }}$ news note, 63

Lessin, Barton $M$., "Issues raised by extended campus services librarians," 14-15

Lelters, 20, 104, 207, 265, 324, 411 $12,453,535-37,639$

Levinson, Anne E., response to letter to the ed. "Rust support evil?" (52:738), comments, 207, 265

"Librarians meet in Budapest," Gyeszly, 374

"Librarians" role on accreditation teams," de Klerk, 535-37

"Librarianship too attractive?" Frank, 411

LAMA, PRS, John Cotton Dana Library Public Relations Award, 1992, 568-70 \& cover; 1993,438 , 570,596

LC, news notes, 373,504

LOEX, news note, 620

lichtenfels, Dave, photo., 280

Lobsien, Bessie Siemens, letter to the ed., 324

Loeb, Linda, "Grants awarded to research libraries to provide national access to resources," 413

La.SU, news notes, 373-74, 438

Lyman, Peter, news note, 278

Lynch, Frances, news note, 604

Lynch, Mary Jo, "Access technology in academic libraries," 243-44

in

McCallum, Brenda, deceased, 737

McCook (Heim) Kathleen de la

Peña, "ALA candidates share their plans for the future ${ }^{n} \&$ port., 249 51

McCoy, Jacquelyn A., ACRL president \& port., 465; "Message from the vice-president" \& port., 651; photos., 382, 524-25, 661

McDonald, Joseph, appt., 735

McKee, Cheryl Spiese "Toward a Bibliothèque Nationale des Arts," 247-48

McNair, Jeanene, deceased, 608

MacNeal Hospital HSRC, John Cotton Dana Library Public Relations Award, 568-70

"Make the most of your student assistants " Condron 5-6

"Making the most of your serials budget," Morton, 630

Malone, Deborah, news note, 734

Mangla, P. B., port., 203

Manning. Leslie A. "Financial report" \& port., 662-64; photos., 524, 661

Marden, Mary Jane, photo., 280

Margolis, Bernard A., "ALA candidates share their plans for the future" \& port., 252

Maricopa Community Coll. District, news note, 60

Marshall, John David, news note, 416

Martinus Nijhoff International West European Specialist Study Grant, ACRL, WESS, 1992, 389; 1993, 529 . 31

Massill, S. W., port., 203

Massman, Virgil F., letter to the ed. \& response $(52: 738)$, comments, 207 , 265
"Media tips:" ("how to get speaking opportunities," Beaubien, 127 ; "speaking successfully," ALA, 182; "what to do when the media calls and some quotes your audience will love," ALA, 326-27)

Mento, Barbara, "Reference roving at Boston College," 634-37

Meszaros, Imre prof, \& port., 215

Metz, Ray E., "ACRL chapters provide forum for electronic information discussions" $16-17$, comment \& response, 265; photos., 524-25, 661

Michalak, Sarah, news note, 674

"Michigan Research Libraries Triangle formed," 504

Mich. State U., "Patron access to onorder and in-process titles, ${ }^{\prime} 702-8$ Micuda, Vladimir, retired, 349

Middendorf, Gertrude Coddington Davis, deceased, 608

Miller, Mary Celine, retired, 737

Miller, Peggy, retired, 419

Miller, William, news note, 132

Minor, Marti A., photo., 128

Miriam Dudley Bibliographic Instruction Librarian of the Year Award, Mountainside Pub. Co., 1992, 279$80 ; 1993,527-28$

Mitchell, Joan, news note, 132

"Model statement for the screening and appointment of academic librarians using a search committee," ACRL, ASC, 642-45

Moffett, William A., news note \& port, 416

Morgan, Eric L., photo., 128

Morris, Leslie R., letter to the ed. \& response, 324

Morton, Donald, "Making the most of your serials budget," 630

Mosher, Paul H., news note, 674

"Motherly care," Charlot, 234 (photo. cover, no. 4)

Mounc Holyoke Coll., news note \& photo., 311

Mountainside Pub. Co., Miriam Dudley Bibliographic Instruction Librarian of the Year Award, 1992, 279$80 ; 1993,527-28$

"Moved to tears," Lobsien, 324

Mudrick, Kristine, news note 734

Murphy, Paula, "Documentation of performance art, ${ }^{n}$ 246-48

Myers, Marcia J, appt. \& port., 605

Natheth, Thomas G., deceased, 608

$\mathrm{NEH}$, news note, 375

Natl. Lib. Week, news notes, 438-39 567

"National Library Week-making it happen," Wallace, 88-89

NREN, "How NREN can meet the needs of academic Jibraries, "705-6

"Need help with your information lit eracy program?" Rader, 718-19

"Need to talk to someone about accreditation?" 448

"Network navigating and navigators," Alldredge, 399-400

"New AV guide available," ALCTS, 278

"New BI book published by ACRL," 274

"New Delhi, India, hosts librarians from around the world, ${ }^{n}$ Ford, 640 "New publications," Eberhart, 54-56 108-12, 205-6, 271-72, 340-41, 409 $10,470-72,599-601,670-71,730-31$ See also Book reviews 
NYPL, news note, 440; "Privare treasures in a public library," 541

Newby, Jill, "A career in BI: dead end or stepping stone? ? $^{n} 178$

News from the field, 59-62, 125-30, 209-14, 273-75, 309-11, 373-75, 437$40,501-4,565-67,628-30,694-96$

Ng'ang'a, James, port., 203

NCSU, "Research materials: now only keystrokes away," 128; "Ten things you can do to help in the scholarly communication crisis," 582

"Nothing ventured, no one gained," Beaubien (52:640-41), comment, 207

Nowell, Fay, deceased, 284

\section{o}

Oberg, Larry R., "The Très grande bibliothèue and the library of the future," 394-96

Oberly (Eunice Rockwell) Award, ACRL, STS, 1993, 311, 532

"The objects of his devotion," Selth (52:712-15), comment, 104

Ochs, Michael, news note, 346

OCLC, "Is FirstSearch really attrac tive?" 461-64; news notes, 210, 565; "UC tries FirstSearch," 464

Off-Campus Library Services Conference, news note, 567

Ohio U., news note, 694

Olivier, Evelyn Riché, "Public relations winners celehrate collections, catalogs, services, and more," 568 70

"Optical Jocal area network at Walla Walla College," Gaskell, 112

"Optical scanning in an academic library," Willett, 698-701

"Or too difficult to achieve?" Bratton, 411

Or. State U., "Training newly appointed reference librarians," 8-9; "User statistics for CD-ROM selection and management," 505-6

Owen, Amy, "Welcome to Utah" \& port., 30

\section{P}

Pantuhoff, Oleg O., "Private treasures in a public library," 541

Pantzer, Katharine, retired, 134

Papers solicited, $60,438,567$ (2), 629 (2)

Parish, David W, retired, 349

Park, Joe, deceased, 284

Pasadena City Coll. (Calif.), news note, 374

Patrick, Sally M., "Utah Library Association annual conference welcomes ACRL," 102-3

"Patron access to on-order and inprocess titles," Ten Have, 702-8

Peischl, Thomas M., photo., 525

Penniman, W. David, ports., 32, 377

Pa.LA news note, $209-10$

People, 63-65, 131-34, 215-18

People in the news, 63, 131-32, 215$17,278-84,345-50,416-19,475-78$, $542-45,604-8,674-76,734-37$

"Periodicals in college libraries: are the challenges of rising subscription costs being met?" Kirk, 94-97

Perry, Emma Bradford, appt, 605

Peru, news note, 59-60

Peters, Klaus, "The CologneCleveland librarian exchange ${ }^{n}$ \& photo., 390-93

Peterson, Trudy H., news note, 674

Phillips, Susan, news note, 734
Phipps, Shelley E., photos., 524-25, 661

Pierson, Robert M., letter to the ed., 453

Pinckard, Mara, port., 29

Pollard, William C., retired, 676

Pollock, Luella (Becky), deceased, 350

Poole, Jay Martin, letter to the ed. 412

Popa, Opritsa D., "Journey to Transylvania: the 1991 ALA colloquium on library science," 172-75, comment 324

Popko, John, news note, 674

Popular Culture Assn., news note, 438

"A potpourri of practical ideas from ACRL's 6th National Conference," 455-60

"Preparing to be a science librarian in college, "Wallin, 171

"Preservation news," Brown (52:649), correction, 6

"Preservation news," Brown, 106, Hedberg, 200, 269, 339, 407, 469, $536,598,668-69,729$

"Preserving the nation's intellectual heritage: a synthesis," Byrne, 31315

Prim, Reggie, acknowledgment, 236; "CERL news staff member has a winning idea," 89

Pritchard, Sarah M. appt. \& port. 476

PALNI, news note, 694

"Private treasures in a public library," Goldsmith, 541

"Products available for Black history month," ALA, 60-61

Profiles, 63, 131, 215

"Promoting careers in library and information science," Vaughters, 38689

"Public relations winners celebrate collections, catalogs, services, and more," Olivier, 568-70

"Publications," Eberhart (52:656) comment, 20

"Publications," Eberhart, 54-56, 108$12,205-6,271-72,340-41,409-10$ 470-72, 599-601, 670-71, 730-31. See also Book reviews

"Put CERL news on your mailing list," $101,175,408$

Quick, Richard C., retired, 349

$\mathbf{R}$

"Racial \& ethnic diversity: information exchange," Hinojosa, 270, 338, 537,669

Rader, Hannelore B., "The CologneCleveland librarian exchange" \& photo., 390-93; "Need help with your information literacy program?" 718-19; "We must be a part of international librarianship, "242-44

Ranganathan, S. R., centennial year $\&$ port., 502

Rare books \& manuscripts librarian ship, "Recruitment open," 183

Rare Books and Manuscripts Librari anship Award, Christie, Manson \& Woods, 1993, 532

Rather, Lucia Johnson, news note 542

"RBMS preconference to be in Santa Cruz," ACRL, 310

"Reaching out to the media," ALA, 259
"Read any good (suggestion) books lately?" Altschiller, 92-93

"Recruiting the best and the brightest" \& port., Beaubien, 320-23

"Recruitment \& retention in your own backyard," Kreitz, 237-40, comments, 411

"Recruitment open for two ACRL editorships," 183

"Reference roving at Boston College," Bregman, 634-37

"Reflections on my recruitment," Tavill, 388

Reichel, Mary, appt. \& port., 605

Reid, Thomas G., Jr., letter to the ed. 207, comment, 265

"Repetitive-motion injuries, ${ }^{n}$ Crampon, 412

"Request forms for extended campus library services," Baker, 260-61

"Research and academic librarians: a global view" \& ports., Shama, 203

Research forum, 94-97

Research forum ("Periodicals in college libraries," Kirk, 94-97)

RLG, news note, 696

"Research materials: now only keystrokes away," Casorso, 128

"Rethinking the librarian's role on accrediting teams," Wolff, 450-51, comment, 535-37

Retirements, 65, 133-34, 218, 284 $348-50,418-19,478,544-45,607-8$ 676,737

Rettig, James, rev. (The whole libram bandbook), 57

"Revitalize library exhibits," UCLA, URL, 125

Rhine, Leonard, "Using a checklist for library staff orientation and training," 695

Ricks, Thomas W., "Coping with repetitive-motion injuries in a large academic library," 161-62, comment, 412; "What, exactly, is carpal cunnel syndrome?" 164-65, comment, 412

RMG Consultants, news note, 629

Rodgers, Frank, news note, 132

"The role of academic libraries in teaching and learning," Adams, 442-45

Rosary Coll., news note, 630

Rosenthal, Joseph A., news note, 132. retired, 134

Rothe, Kurt, news note, 734

Rudolph, Ellen, retired, 478

"Rust debate continues," Reid, 207 comment, 265

"Rust support evil?" Massman \& response (52:738), comments, 207 , 265

Rutgers U., news note \& drawing, 59 news notes, 310,374

Ryan, Mary, retired, 65

Saffo, Paul, photo., 376; port., 32

Sager, Rochelle, photos, , 524-25, 661

St. Petersburg Jr. Coll., "Collection development program recognized" \& phoro., 280

Salisbury, Lutishoor, "Is FirstSearch really attractive?" 461-64

"Salt Lake City-culture and entertainment," Hall, 100-101

"Salt Lake City-restaurants and clubs to enjoy," Hall, 186-87

Samuel Lazerow Fellowship, ISI 1992,$346 ; 1993,531$

Sanders, Kathy, "University of Arkan 
sas at Little Rock institutes student library fee," 439

Saur (K. G.) Award for Best College $\varepsilon$ Research Libraries Article, 1992, $346 ; 1993,532$

Savage, Daniel A, news note, 674

Schaeffer, Reiner H. "Swiss," It. Col, retired, 737

Scherdin, Mary Jane, appt. \& port. 675

Schick, Frank L., deceased, 284

Schimmel, Herbert D., letter to the ed. 104

Schoonover, Phyllis J., retired, 419

Scors, Taisa, deceased, 608

Scott, Mark, letter to the ed., 453

Scott, Shirley R., "User statistics for CD-ROM selection and manage ment, "505-6

Seal, Robert, news note, 674

Segal, Judith, appt., 735

Selth, Jeff, "The objects of his devotion" (52:712-15), comment, 104

Sessions, Judith A., "ALA candidates share their plans for the future" \& port., 252-54; appt., 282

"Share your library's news," CERL news, 447

"Share your networking stories," 399

"Share your opinion with CERI neus readers," $14,97,174$

Sharma, R. N., "Books are for use Ranganathan centennial year," 502 "Research and academic librarians a global view" \& ports., 203

Sheehan, Jelka, retired, 134

Sheehy, Carolyn, news note 475

"Shifting paradigms for librarians," Hubbard, 707-8

Shipps, Tony, retired, 478

Shishido, Tsuyuko, news note, 542

Simmons, Howard, interview \& port. 442-45

Sims, Roberta Laulicht, "Beyond the book review, ${ }^{\text {}} 646$

Smith, Alice Reviere, "The CologneCleveland librarian exchange ${ }^{n}$ \& photo., 390-93

Smith, K. Wayne, news note, 542

Smith, Wilbur Jordan, deceased, $\mathbf{4 1 9}$

SPIE, news note, 309-10

Southern Methodist U., news note, 210

Speiden, R. Virginia, deceased, 134

"Spellcheckers must be checked," Harper, 567

Spiegel. Pam, acknowledgment, 236

"Spring and diversity in the air at ACRL conference," 376-85

Stam, David $H_{.}$, news note, 674

"Standards for faculty status for college and university librarians," ACRL, ASC, 317-18, comment, 453

Stanford U., Leab award, 281; news note, 209

Starratt, Jay, appt. \& port., 347

State, Mary Ellen, retired, 349

SUNY (Binghamton, "Optical scanning in an academic library," 698 701: Buffalo, news note, 310-11; U at Albany, news note, 60)

Stetson U., news note \& photo., 273 74

Stevens, Norman D., "ALA candidates share their plans for the future" \& port., 251-52

Stillwell, Stephen J., Jr., news note, 674

Stimpson, Catharine R., photo., 377 ; port., 32

Stoffle, Carla, "ACRL honors the best in academic and research librarianship" \& port., 184; photo., 528

Strauch, Katina P., news note, 542-43

Sullivan, Pegry, "Named ALA executive director" \& port., 437

Summit, Roger K., news note, 132

Swank, Kristine, news note, 475

Sweet, Don, "University of Arkansas at Little Rock institutes student library fee," 439

Symons, Ann, "ALA candidates share their plans for the future" \& port., 254

Tabb, Winston, prof, \& port., 131

Taiwan, Natl. Central Lib., news note, 210-12

Tamblyn, Eldon W., acknowledg ment, 84

Tatian, Carol, news note, 345

Tavill, Kay, "Reflections on my recruitment," 388

Taylor, Colin, port., 203

"Ten things you can do to help in the scholarly communication crisis, "NCSU, 582

Ten Have, Elizabeth, "Patron access to on-order and in-process titles," $702-8$

Tex. A\&M U., "Coping with repetitive-motion injuries in a large academic library," 161-62, comment, 412; "Fun, friends, and good p.r. celebrating National Library Week in an academic library," 85-89

Tex. Tech, news note, 273

Tex. Woman's U., "Make the most of your student assistants," 5-6 "Think before you Post-it"

Thomas, Bruce E., retired, 65

Thomas, Mary Ellen, retired, 65

Thomas, Robert D., news note, 63

Thompson, Donald, deceased, 419

Thompson, Dot S., "Beyond the book review, ${ }_{1} 646$

Timberlake, Clare $\mathrm{H}$., port, 126

"The time to lead," ACRL, 256-57

"Tips for librarians planning for accreditation visits," 447

Tolson, Stephanie D., appt., 347

"Toward a Bibliothèque Nationale des Arts," McKee, 247-48

"Training newly appointed reference librarians," Wogaman, 8-9

Traylor, Bill, "University of Arkansas at Little Rock institutes student library fee," 439

"The Très grande bibliothèque and the library of the future," Oberg, 394-96

Trout, Frank, retired, 134

"Two million reasons to celebrate," UC, SB, cover, no. 9

$\mathbf{U}$

USIA, "April 15 deadline for library/ book fellows," 274

U. of Alta., Leab award, 281

U. of Ariz, news notes, 375, 440

U. of Ark., Fayetteville, "Is FirstSearch really attractive?" 461-64

"University of Arkansas at Little Rock institutes student library fee," Sweet, 439

UC, news note, 209 (LA URL, news note, 125; Riverside [news note, 605-96; "The objects of his devotion" (52:712-15), comment, 104]: San Diego, news note, 125; Santa Barbara ["Collecting primary materi- als of major ethnic groups," 157-59; John Cotton Dana Award, 568-70 (Book of bours, photo., cover, no. 9)]

"UC tries FirstSearch," Crooks, 464

U. of Central Fla., "About the cover," 626 (photo., cover, no. 10)

UC, Denver, "About the cover," 690 (photo., cover, no. 11)

U. of Del., news note, 375

U. of Fla., HSC, "Using a checklist for library staff orientation and training," 695

UI (Chicago, news note, 504; U-C Inews note, 438; "When are library records confidential?" 129 , clarification, 276])

U. of Kan., news note, 438

U. of $\mathrm{Md}$. Leab Award, 281 (Coll Park, news note, 210)

U. of Mass. Medical Ctr., "Making the most of your serials budget," 630

U. of Mich., news notes, 309, 694

UNC, Charlotte, news note, 59

U of Notre Dame, news note 694

U. of Pittsburgh, news note, 210

U. of S. C., news note, 210

U. of Southern Calif, news note, 210 12

U. of Tenn., Martin, news note, 309

UT, Austin, "The future of reference IV" 508-14; news note, 59

U. of Toronto, Leab award, 281

$\mathrm{U}$. of $\mathrm{Va}$, Leab award, 281

U. of Wash., news notes, 311,375

"User statistics for CD-ROM selection and management," Butcher, 505-6

Usher, Caroline, letter to the ed, 207

"Using a checklist for library staff orientation and training," Rhine, 695

"Using White House Conference recommendations, ${ }^{n}$ Wand, 22, 25

Utah Lib. Assn., "Conference runs concurrently with ACRL National Conference," 33

"Utah Library Association annual con ference welcomes ACRI," Patrick $102-3$

V

Valauskas, Edward, photo., 173

Vanderbilt $U_{\text {, , news note, } 438}$

Van Deventer, Barbara, "Preserving the nation's intellectual heritage: a synthesis," 313-15

Vaughters, Sharon, "Promoting careers in library and information science," 386-89

Vazakas, Susan M., "Where are all the science librarians?" 166-71

Veatch, Van, retired, 134

Viera Ann letter to the ed, 104

"Vista de S. Francisco," Ferran, 306 (photo., cover, no. 5)

\section{$N, x$}

Wagner, Elaine, "Emory Lniversity Libraries' Environmental Action Force," 579-80

Walla Walla Coll. "Optical local area network at Walla Walla College," 112

Wallace, Linda K., "National Library Week-making it happen," 88-89

Wallin, Camille Clark, "Preparing to be a science librarian in college," 171 ; "Where are all the science librarians?" 166-71

Wand, Patricia A., "How NREN can meet the needs of academic libraries," 705-6; "Using White House 
Conference recommendations," 22 , 25; ed., "WHCLIS teleconference stimulates action plan for Colorado," 105

"War is not exciting," Bradley, 20

"Washington hotline" Henderson, $24-25$, 189-90, 267-69 (comment, 453), 337, 405, 468-74, 534, 597$603,666-69,728$

The way I see it $(52: 711)$, comment, 104

The way I see it, 242-44, 320-23, 45051 (comment, 535-37), 581-82, 7056

The way I see it ("Food for thought," Gater [52:711], comment, 104)

The way I see it ("How NREN can meet the needs of academic libraries," Wand, 705-6; "Recruiting the best and the brightest," Beaubien, 320-23; "Rethinking the librarian's role on accrediting teams," Wolff, 450-51, comment, 535-37; "We must be a part of international libratianship," Rader, 242-44; "What if we viewed library instruction like driver ed?" Bain, 581-82)

"We must be a part of international librarianship," Rader, 242-44

Weiss, Bernice, retired, 218

"Welcome to Utah" \& port., Owen, 30

Welk, Lawrence, port., 540

Wells, Margaret, appt., 605
Western Ky. U., "Request forms for extended campus library services," 260-61

Western Ky. U., Women's Studies Conf., "Call for papers," 60

WLN, news note, 309

"What, exactly, is carpal tunnel syndrome?" Ricks, 164-65, comment, 412

"What if we viewed library instruction like driver ed?" Bain, 581-82

"When are library records confidential?" UI, U-C, 129 , clarification, 276

"Where are all the science librarians?" Vazakas, 166-71

"Where are the jobs?" Usher, 207

White, Kris A., news note, 604

WHCLIS, "Using White House Conference recommendations," 22, 25 ; "WHCLIS teleconference stimulates action plan for Colorado," 105

"WHCLIS teleconference stimulates action plan for Colorado," Alire, 105

The whole library bandbook, Eberhatr, rev. of, 57

Whole person catalog 2, news note, 275

Willett, Perry, "Optical scanning in an academic library, ${ }^{, 698-701}$

Williams, Delmus, appt., 347

Wilson, Betsy, appt. \& port., 282-83

Wilson, Carolyn T., news note, 416

Wilson (H. W.) Co., John Cotton
Dana Library Public Relations Award, 1992, 568-70 \& cover; $1993,438,570,596$

Winnegrad, Mark H., letter to the ed., 639

Wittkopf, Barbara J., "Kirk and Wittkopf share their visions for ACRL" \& port., 194-99; photos., 524-25, 661

"Witty suggestion book comments sought, "CERL news, 93

Wogaman, Mariol R., "Training newly appointed reference librarians, " 8-9

Wolff, Ralph A., "Rethinking the librarian's role on accrediting teams, " 450-51, comment, 535-37

Women's studies collection development policies, ACRL, 375

Wood, Richard J., news note, 674

Woodall, Nancy C., letter to the ed., 535

\section{$\mathbf{Y}$}

Yandle, Anne, retired, 349-50

Yates, Stanley, retired, 65

Young, Phillip H., "Death of the book greatly exaggerated," 452-53, comment 535

"Your responsibilities as a media spokesperson," ALA, 408

$\mathbf{z}$

Zager, Daniel, news note, 282

\section{- ACRL Approval Plans ... Save Time and Money! \\ ACRL Publications Provide Practical Ideas, Tools and Methods for: COLLEGE \\ - Management - Bibliographic Instruction - Special Collections \& RESEARCH - Collection Development - Statistics \& Research \\ The convenient and cost-cutting ACRL approval order plan provides automatic priority shipping of ACRL's new books at a $20 \%$ discount to ACRL members ( $10 \%$ to non-members). \\ There are two approval plan categories from which to choose: \\ Plan $\mathbf{P}$ guarantees that you will automatically be sent all new ACRL publications" including CLIP Notes. ACRL publishes approximately 5 to 6 new titles each year. \\ -Periodicals and subscription items are not included. \\ You may change or cancel your approval order at any time. Retum privileges guaranteed \\ Enroliment Form \\ a Plan $\mathrm{P}$ guarantees shipment of all new non-periodical $\mathrm{ACRL}$. \\ a Plan PC guarantees shipment of all new CLIP Notes. publications including CLIP Notes. \\ Please give your ALA membership number if you are eligible for the $20 \%$ ACRL membership discount. \\ Note: Personal memberships may not be used to qualify institutions for the ACRL member discount. \\ ALA membership number \\ P.O. $=$ \\ Ship to: \\ Bill to: \\ Return this form to: \\ ACRL Approval Order Plans ACRL/ALA \\ 50 East Huron Street \\ Chicago, IL 60611-2795 \\ Or call: \\ $1(800) 545-2433$}




\section{H O I C E}

\section{ETHNIC STUDIES REVIEWS SERIES}

CHOICE announces a new series of bibliographies that identifies scholarly books covering a number of ethnic groups.

The Ethnic Studies Reviews series has been designed to facilitate collection development in several multicultural areas, to provide current bibliographies on particular ethnic groups, and to facilitate research and reference.

The bibliographies will benefit faculty and students, as well as librarians, by providing a readable list of books published in the last three or four years accompanied by a full review, plus an author and title index. Finding a recent publication on or about a particular ethnic group and developing a course or reading list can be accomplished easily with these new $\mathrm{CHOICE}$ bibliographies.

Each bibliography addresses a particular American ethnic group and is compiled from favorable reviews previously published in CHOICE. The titles cross all disciplines and represent a selected view of the best scholarly titles published on one of these multcultural areas.
The first titles in the new series:

African and African American

Studies; 146 pages; 754 titles; $\$ 22.00$

Latino Studies; 100 pages;

531 titles; $\$ 20.00$

Native American Studies;

54 pages; 278 titles; $\$ 15.00$

Plus $\$ 2.00$ postage and handling for each title ordered.

Forthcoming:

Asian and Asian American Studies, in 1993

Order one or all of these bibliographies directly from:

\section{CHOICE}

100 Riverview Center

Middletown, CT 06457

CHOICE

Current Reviews for Academic Libraries 